\title{
INVESTIGACIONES ARQUEOLÓGICAS EN LA CUENCA DEL ARROYO CHASICÓ Y LLANURA ADYACENTE, SUDOESTE DE LA PROVINCIA DE BUENOS AIRES
}

\author{
Fernando Oliva, ${ }^{*}$ Juan David Avila, ${ }^{* *}$ María Pía Ayuso, ${ }^{* *}$ \\ Luciana Catella*** y Mariela Gallego**** \\ ${ }^{*}$ CEAR, FHumyAr, UNR. FCNyM, UNLP. \\ ** Becarios CONICET. CEAR, FHumyAr, UNR. \\ *** Becaria UNLP. FCNyM, UNLP. CEAR, FHumyAr, UNR. \\ **** Becaria CIC. FCNyM, UNLP. CEAR, FHumyAr, UNR.
}

\begin{abstract}
Resumen
En este trabajo se presenta la información generada a partir de prospecciones y recolecciones superficiales llevadas a cabo en la cuenca media e inferior del arroyo Chasicó y en el área que conforma su limite Oeste. Las mismas tuvieron por objetivo reconocer la disponibilidad de recursos en los diferentes sectores de la llanura adyacente al Sistema Serrano de Ventania y analizar las vinculaciones establecidas entre los distintos ambientes del área, considerándose en particular en esta primera etapa, el material lítico, por ser un recurso que brinda información sobre el uso del espacio y las vinculaciones existentes entre los diferentes ambientes.
\end{abstract}

Palabras Claves: Cuenca Arroyo Chasicó, cazadores- recolectores, ambiente ecotonal, recursos líticos.

\begin{abstract}
In this paper, new archaeological data is presented as a result of several surveys carried out and surface materials collected from the middle and lower Arroyo Chasicó basin and along the western border of that region. The main goal of that fieldwork was to establish the availability of resources in the various environments adjacent to the Sistema Serrano de Ventania. In this preliminary stage of analysis, the interest was focused upon lithic materials, because its inherent properties to yield information on links between different ecological regions and the use of space by hunting and gathering societies.
\end{abstract}

Key Words: Chasicó Stream Basin, hunter-gatherers, ecotonal environment, lithic resources.

\section{INTRODUCCIÓN}

El estudio de las sociedades cazadoras recolectoras que habitaron la Región Pampeana se inicia hacia fines del siglo XIX, abordando a lo largo de su historia y desde diferentes perspectivas teóricas, temas relacionados con el uso, manejo y ocupación de los distintos ambientes presentes en esa región (p.ej. Ameghino 1910; Hrdlicka 1912; Menghin y Bórmida 1950; Austral 1968; Politis 1984; Frère y González de Bonaveri 1993; Berón et al. 1995; Barrientos et al. 1997; Oliva y Moirano 2001; Bayón et al. 1999; Oliva 2000; Madrid y Politis 2001; Mazzanti y Quintana 2001; Aguerre 2002; Martínez 2002; Paleo et al. 2002; Oliva et al. 2004).

Desde una perspectiva ecológica, el área de estudio se caracteriza por estar emplazada en el extremo Sur de la Región Pampeana, limitando con el Norte de Patagonia, en ambientes ecotonales entre las provincias fitogeográficas Pampeana y del Espinal. Esta ubicación particular posibilita la presencia de especies vegetales y animales propias de cada región. Dicha característica, sumada a la presencia de agua y recursos minerales localizados, convierten al área en un espacio atractivo, donde las sociedades cazadoras recolectoras debieron emplear diversas estrategias vinculadas con la toma de decisiones respecto al 


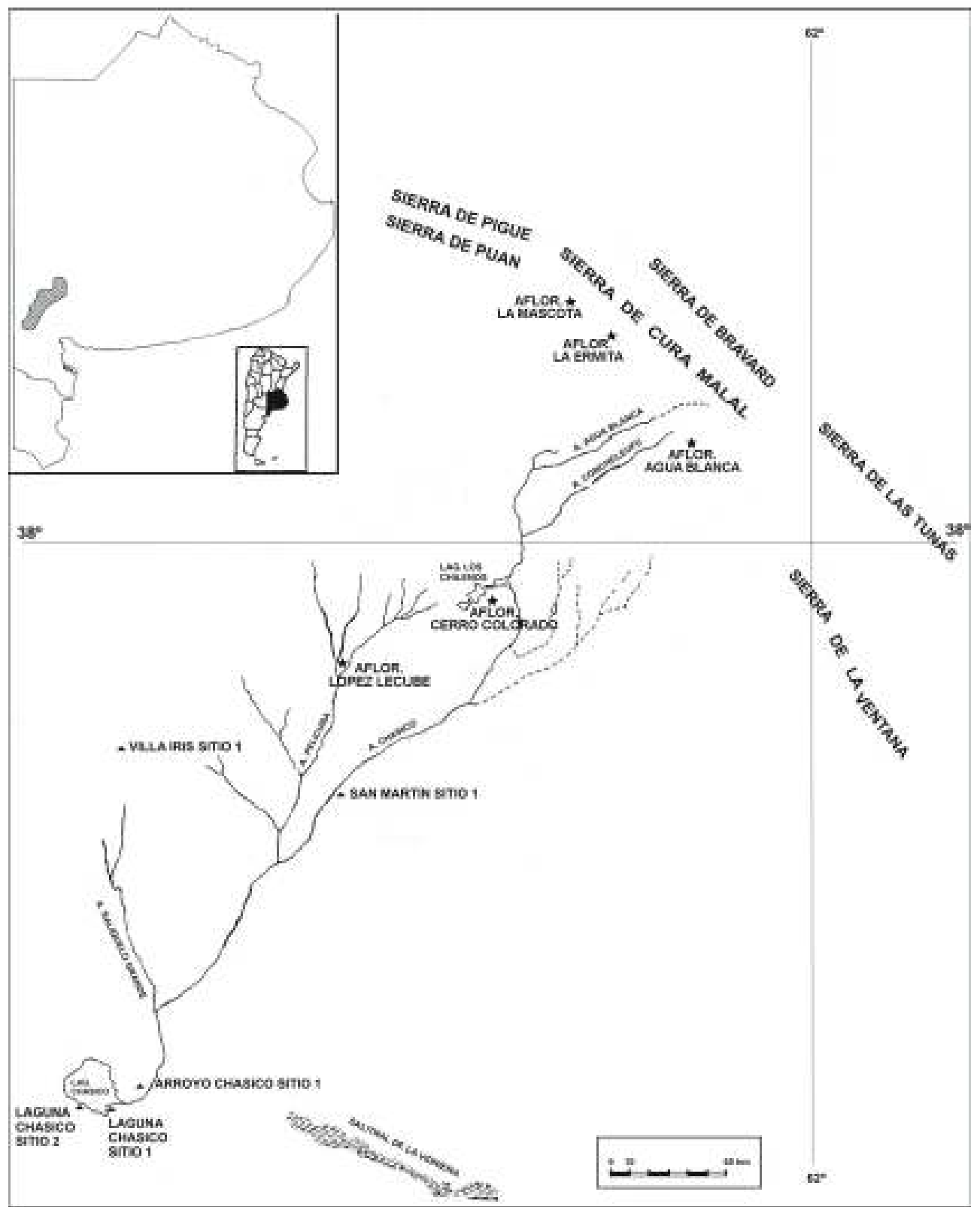

Figura No 1 Mapa de la región con la ubicación de los sitios afloramientos rocosos y principales accidentes geográficos

manejo, procesamiento y adquisición de la información (Buckley 1978; Mithen 1990). De esta ecotonales entre las provincias fitogeográficas Pampeana y del Espinal. Esta ubicación particular posibilita la presencia de especies vegetales y animales propias de cada región. Dicha característica, sumada a la presencia de agua y recursos minerales localizados, convierten al área en un espacio atractivo, donde las sociedades cazadoras recolectoras debieron emplear diversas estrategias vinculadas con la toma de decisiones respecto al manejo, procesamiento y adquisición de la información (Buckley 1978; Mithen 1990). De esta manera, se considera oportuno introducir la propuesta de Mithen (1990) en cuanto a la noción de "meta-decision making". Este concepto estaría comprendiendo la más austera 
decisión que se puede tomar. En este sentido es esencial la "decisión" en el momento de adquirir información, considerando qué tipo de información resulta fundamental para resolver un problema en particular, las variables alternativas que se encuentran disponibles, qué conocimientos particulares se tienen de ellas, qué es lo que se necesita conocer, cuáles son los caminos en la obtención de esta información y de estos caminos cuáles son más relevantes. Al mismo tiempo se debe considerar por qué es necesario adquirir ese particular tipo de información y cuáles son las reglas del conocimiento disponibles escogiendo las que deben ser usadas. En síntesis, la "meta-decision making" trae aparejado considerar todo el universo disponible en la relación de las sociedades con el medio así como conceptos ecopsicológicos (Mithen 1990).

Con el fin de analizar el empleo de ciertos recursos críticos, y establecer vinculaciones entre los distintos sitios del área, se considera en particular el material lítico, por ser un recurso que brinda información sobre la toma de decisiones en el uso del espacio y las vinculaciones existentes entre los diferentes ambientes.

En este trabajo se presenta la información generada a partir de prospecciones y recolecciones superficiales llevadas a cabo en la cuenca media e inferior del arroyo Chasicó y en el área que conforma el límite Oeste de la misma. Por medio de estas actividades se retomaron las investigaciones desarrolladas a principios de la década del '90 por uno de nosotros (F.O.) en San Martín sitio 1 -Partido de Puan- y Laguna Chasicó sitio1 -Partido de Villarino-, y se identificaron tres nuevos sitios arqueológicos: Arroyo Chasicó sitio 1 -Partido de Villarino-, Villa Iris sitio 1 y Laguna Chasicó sitio 2 -Partido de Puan- (Figura $N^{\circ} 1$ ).

\section{ANTECEDENTES}

En el área serrana de Ventania y su llanura adyacente, las investigaciones se inician con los trabajos de Holmberg a fines del siglo XIX (Holmberg 1884), y se continúan a partir de la década del '60 con los aportes de diferentes investigadores (entre otros Austral 1968, Madrazo 1979, Gradin 1978, Castro 1983 y Pérez Amat et al. 1985).

Hacia fines de la década del ' 80 se consolidan las investigaciones sistemáticas en este área de estudio, posibilitando la obtención de información arqueológica proveniente de sitios en superficie y en posición estratigráfica (entre otros, Oliva et al. 1991 a y b; Barrientos et al. 1997 y 2002; Oliva et al. 2000). Al mismo tiempo se inician trabajos intensivos en aleros y cuevas con representaciones rupestres (entre otros, Madrid y Oliva 1994; Consens y Oliva 1999; Oliva 2000; Oliva y Algrain 2004).

Específicamente en la cuenca del arroyo Chasicó, se realizaron investigaciones en la laguna Los Chilenos -sitios 1 y 2- (Barrientos et al. 1997 y 2002), en la localidad La Montaña -sitios 1, 2 y 3- (Oliva 2000; Oliva et al. 2000), como así también sobre la margen izquierda del arroyo Chasicó -San Martín sitio 1- (Oliva et al. 1991a; Oliva 1996). En este último se han recuperado materiales en posición estratigráfica asignados a inicios del Holoceno Tardío, entre los que se pueden mencionar: artefactos líticos confeccionados sobre diversas materias primas asociados a restos faunísticos, pigmentos, cuentas de collar confeccionadas en valva, y caracoles marinos (Adelomelon brasiliana). Estos materiales estarían informando sobre una ocupación de actividades específicas vinculadas con el procesamiento de material faunístico. Cabe destacar en este sitio la presencia de Lestodelphys halli, marsupial que en la actualidad 
sólo habita en zonas más áridas, lo cual estaría indicando cambios ambientales en la región a finales del Holoceno Medio (Oliva et al. 1991a; Oliva 1996).

Por último, se realizaron prospecciones en varios puntos de los márgenes de la laguna Chasicó y en los médanos adyacentes, identificándose hallazgos aislados y concentraciones de material. Entre los artefactos identificados se encuentran elementos de molienda e instrumentos tallados sobre rodados tehuelches (sensu Fidalgo et al. 1978), cuarcita y riolita, entre otras materias primas (Oliva 1996).

\section{CARACTERÍSTICAS AMBIENTALES}

Fitogeográficamente, la cuenca del Arroyo Chasicó incluye las provincias Pampeana Distrito Pampeano Austral- y del Espinal -Distrito del Caldén- (Cabrera 1971). Se inicia en el área de ecotono entre ambas provincias, y la cruza de forma transversal, hasta llegar plenamente a la Provincia del Espinal en su desembocadura.

Se trata de un sistema endorreico cuyas nacientes se ubican en la Sierra de Curamalal, en el sector occidental del Sistema Serrano de Ventania y que tiene su desembocadura en la laguna Chasicó, comprendiendo los partidos de Saavedra, Puan, Tornquist y Villarino en la Provincia de Buenos Aires. Esta última se encuentra ubicada a $120 \mathrm{Km}$. al Sur del Sistema Serrano de Ventania y a unos $100 \mathrm{Km}$. al Noroeste de la costa Atlántica, siendo su nivel de base de $20 \mathrm{mbnm}$ (Bonorino et al. 1989). Tal particularidad permite la captación del escurrimiento superficial circundante, razón por la cual probablemente haya contado con agua inclusive durante períodos de sequías regionales.

Hacia el Oeste de esta cuenca fluvial se concentran una serie de cuerpos lagunares temporarios y permanentes de diferentes dimensiones. Entre las lagunas de tamaños menores se encuentra la laguna Villa Iris, donde se localizó el sitio 1.

En el Sur del Área Ecotonal Húmeda-Seca Pampeana, categoría recientemente definida por Oliva y Algrain (2005), los recursos líticos se sitúan en lugares precisos. Particularmente, en el Sistema Serrano de Ventania afloran rocas cuarcíticas, areniscas y limolitas con diferente grado de silicificación. En este mismo sistema, en el Abra de Saavedra se encuentran los afloramientos de riolita de La Mascota y La Ermita. Los granitos por su parte aparecen puntualmente en la llanura adyacente a este sistema serrano, como López Lecube, Cerro Colorado y Agua Blanca. Por otro lado se localizan fuentes secundarias de materias primas líticas conformadas por los rodados de los cursos de agua (principalmente arroyo Chasicó y sus afluentes, y Río Sauce Chico) y los mantos de rodados tehuelches que se ubican al Sur de la Laguna Chasicó y sobre la costa atlántica (Figura $\mathrm{N}^{\mathrm{o}}$ 1).

Las características mencionadas vuelven al área de estudio un espacio de particular atractivo para las sociedades cazadoras recolectoras. Por otro lado, la utilización de salinas y salitrales, como las Salinas Chicas, ubicadas a menos de $20 \mathrm{Km}$. al oeste de la Laguna Chasicó pudo haber sido otro factor de importancia, dada la recurrente mención en diferentes crónicas de la utilización de la sal por parte de los grupos cazadores recolectores que habitaron la Región Pampeana y Norte de Patagonia (Falkner [1774] 2003, Musters [1911] 2005, Zeballos [1881] 2002). 


\section{CUENCA MEDIA DEL ARROYO CHASICÓ Y ÁREA ADYACENTE A LA MISMA}

\section{San Martín Sitio 1}

Se localiza en la segunda de tres terrazas fluviales, en la margen izquierda del arroyo Chasicó, sobre sedimentos correspondientes al Holoceno Tardío.

En sucesivas investigaciones realizadas en el sitio se llegó a excavar una superficie de 38 $\mathrm{m}^{2}$ (Oliva et al. 1991a; Oliva 1996). Observaciones realizadas en el año 2003 permitieron calibrar el grado de erosión, fundamentalmente hídrica, ocurrida en el mismo. Los perfiles de las cuadrículas excavadas en la década del' 90 se encuentran fuertemente erosionados, lo cual produjo que abundante cantidad de material arqueológico quedara expuesto en superficie. Dada esta situación se trazó un cuadriculado de $225 \mathrm{~m}^{2}$ dividido en 64 unidades, sobre el cual se efectuó la recolección superficial. Se registraron las concentraciones de materiales, así como la topografía del terreno y la vegetación a fin de realizar comparaciones con las plantas relevadas con anterioridad (Figuras $\mathrm{N}^{\circ} 2$ y 3 ).

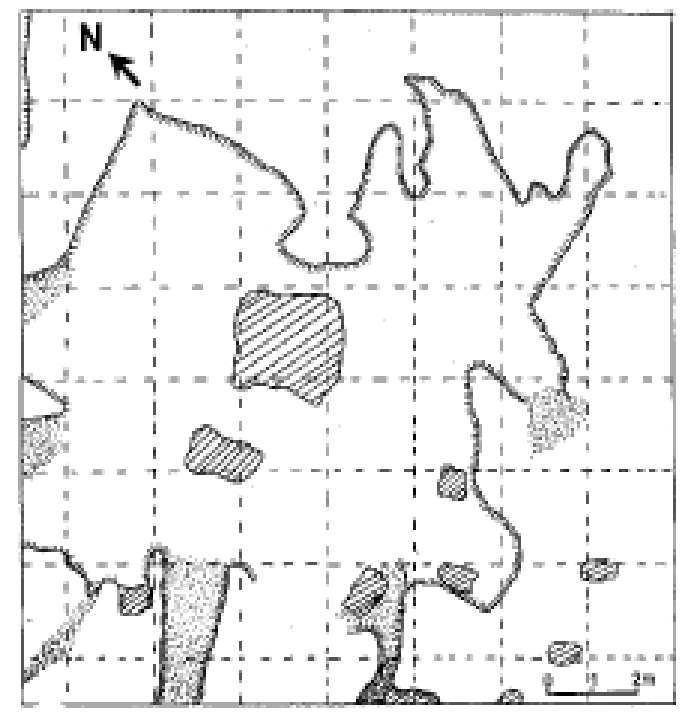

\section{REFERENCIAS}

Fin Liaco do berronca

A Desurend rinets do bersarca

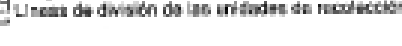

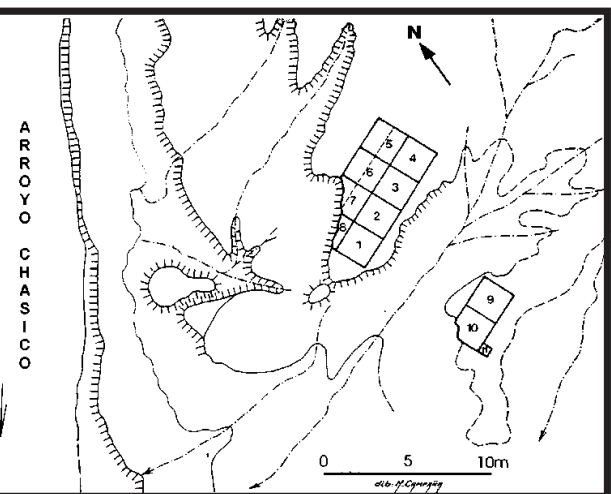

Figura 2. San Martín sitio 1. Planta del sitio donde se observa la topografía del área y las unidades de recoleción realizadas en el año 2003.

Distribuido en estas unidades de recolección se recuperaron cáscaras de huevo de Rhea americana y placas de Dasipodidae (con evidencias de combustión en muchas de ellas), restos óseos faunísticos muy fragmentados y restos de valva de la especie Adelomelon brasiliana. 


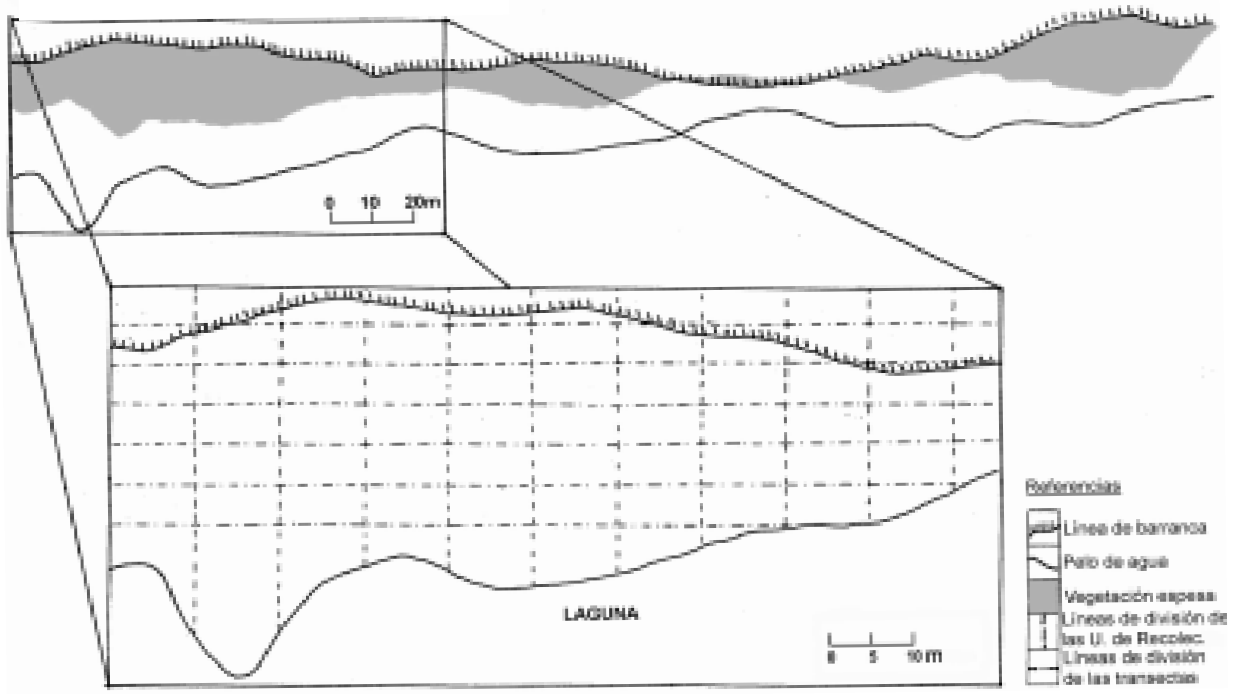

Figura 3. Sitio San Martín 1. planta del sitio deonde se observa la topografía del área y la ubicación de las cuadrículas de excavación en el año 1991

Se analizaron 179 elementos líticos, correspondientes al total de la muestra recuperada en la recolección superficial.

Las principales materias primas observadas fueron cuarcita de diferentes calidades (32\% grano grueso, $41 \%$ grano medio y $12 \%$ grano fino), calcedonia $(4 \%)$, cuarzo $(3 \%)$ y en menores porcentajes riolita $(2 \%)$, chert silíceo $(1 \%)^{1}$ y otras $(5 \%)$.

Las cuarcitas se presentan en todos los tamaños, predominando las de tamaño pequeño y muy pequeño (Tabla $\mathrm{N}^{\circ} 1$ ). La reserva de corteza en los artefactos confeccionados sobre esta materia prima es escasa, prevaleciendo aquellos sin corteza $\left(\right.$ Tabla N $^{\circ}$ 2). Con respecto a los grupos y subgrupos tipológicos representados, se advierte la presencia de núcleos, gran número de $\operatorname{lascas}^{2} \mathrm{y}$ en menor cantidad desechos no clasificables. Entre los escasos instrumentos en cuarcita de la muestra se destacan raspadores, raederas, un fragmento de limbo de punta de proyectil y artefactos de molienda (Tabla $\left.\mathrm{N}^{\mathrm{o}} 3\right)$.

Los artefactos confeccionados sobre calcedonia se encuentran representados por tamaños pequeño y muy pequeño (Tabla $\mathrm{N}^{\circ} 1$ ). Estos artefactos son en su mayoría lascas sin reserva de corteza, siendo los únicos instrumentos dos raspadores con abundante reserva de corteza (Tablas $\mathrm{N}^{\mathrm{o}} 2$ y 3 ).

Entre las materias primas restantes pueden mencionarse riolita, chert silíceo y arenisca, observados en lascas sin corteza de tamaños pequeño, mediano pequeño y mediano grande. El cuarzo se encuentra representado por lascas. A su vez, se destaca el hallazgo de una lasca con rastros complementarios en uno de sus filos, confeccionada sobre obsidiana, de tamaño pequeño y sin corteza. 


\section{Villa Iris Sitio 1}

Se encuentra localizado en la margen Oeste de una laguna de pequeñas dimensiones, ubicada en las inmediaciones de la localidad de Villa Iris, Partido de Puan. En esta laguna se realizó un reconocimiento de toda la línea de costa, identificándose dos concentraciones de material arqueológico y varios hallazgos aislados.

Se efectuó el relevamiento topográfico de la costa de la laguna donde se observó la mayor de estas concentraciones, denominada sitio 1 , y se realizó una recolección superficial sistemática de los materiales localizados en los primeros $100 \mathrm{~m}$. de la misma. Para esta actividad se efectuaron sobre la margen Oeste de la laguna 10 unidades de recolección de $10 \mathrm{~m}$. de largo, divididas en 6 transectas de $2 \mathrm{~m}$. de ancho, ubicadas entre el pelo de agua y la pequeña barranca.

A partir de estas recolecciones se recuperaron restos óseos faunísticos muy fragmentados y dispersos en todas las unidades de recolección, cáscaras de huevo de Rhea americana, placas de Dasipodidae, 2 cuentas de collar, fragmentos cerámicos y material lítico.

La muestra de materiales líticos considerada para este trabajo corresponde a las tres primeras unidades de recolección, llegando a un $\mathrm{N}=384$ elementos. En esta muestra predominan las cuarcitas con el $23 \%$ (cuarcita grano fino 3\%, mediano $12 \%$ y grueso $8 \%$ ) y chert silíceo $(22 \%)$. En tercer lugar se ubican calcedonia (13\%), otros sílices (13\%) y riolita $(13 \%)$. El $16 \%$ restante esta integrado por pequeñas proporciones de otras materias primas tales como hematita, limolita, cuarzo y arenisca entre otras.

Los artefactos confeccionados sobre sílice (calcedonia, chert silíceo y otras variedades de sílice) se caracterizan por ser en su mayoría de tamaños pequeños, presentándose en tamaño mediano grande únicamente aquellos realizados en chert silíceo (Tabla $\left.\mathrm{N}^{\circ} 4\right)$. Entre los silices predominan las lascas, hallándose también algunos instrumentos: raspadores, artefactos de formatización sumaria, raederas (confeccionadas sobre chert silíceo principalmente), y 2 puntas de proyectil, la mayor parte sin reserva de corteza (Tablas $\mathrm{N}^{\circ} 5$ y 6 ).

Del análisis efectuado sobre las cuarcitas se observa la presencia de artefactos en todos los tamaños, predominando levemente los de tamaño pequeño. El $48 \%$ presenta corteza en diferentes proporciones. Con respecto a los grupos tipológicos predominan las lascas y desechos de talla no clasificables, hallándose también algunos núcleos y artefactos de formatización sumaria.

Sobre riolita se encuentran representadas lascas y desechos de talla no clasificables, en tamaños pequeño y muy pequeño, todos ellos sin reserva de corteza.

Entre las restantes materias primas pueden mencionarse: arenisca, hematita, cuarzo, granito, limolita y otras. Estas materias primas se encuentran representadas principalmente por lascas $(4 \%)$, desechos de talla no clasificables $(5 \%)$ y pigmentos no preparados -hematita$(2,5 \%)$; en tamaños pequeños $(10 \%)$ y algunos de ellos en tamaños grandes $(2 \%)$.

\section{Cuenca inferior del Arroyo Chasicó}

Otros sitios arqueológicos considerados para este análisis se localizan en la laguna Chasicó así como en la desembocadura del arroyo homónimo. Esta laguna, como ya se ha indicado, 
se ubica en un ambiente de características particulares dentro de la Provincia del Espinal, a menos de $100 \mathrm{Km}$. del ecotono actual con la Provincia Pampeana; lo que posibilita el desarrollo de abundantes recursos entre los que pueden mencionarse: animales, vegetales, agua y materias primas líticas. Otra característica importante es la localización de salinas cercanas a la laguna, donde las sociedades cazadoras recolectoras pudieron aprovisionarse de sal, recurso mineral muy utilizado por las mismas (Falkner [1774] 2003, Musters [1911] 2005, Zeballos [1881] 2002).

Por otro lado, el área presenta fuentes secundarias de materias primas líticas representadas por remanentes de "rodados tehuelches" del Norte de Patagonia (sensu Fidalgo et al. 1978), y por rodados principalmente cuarcíticos provenientes de Ventania, acarreados por el Arroyo Chasicó.

Estas características llevaron a retomar las investigaciones comenzadas por uno de los autores (F. O.), realizándose en el mes de septiembre de 2003 el relevamiento ambiental de la zona y la identificación de nuevos sitios arqueológicos. A partir de este relevamiento pudo observarse en primer lugar que la línea de costa avanzó sobre la localidad de Chapalcó, inundando la mayor parte de la misma. Otro punto donde pudo identificarse esta inundación fue en la desembocadura del arroyo, la cual se presenta desdibujada y con numerosas islas que con anterioridad conformaron la costa del arroyo. En este sector de la cuenca se observan numerosos recursos pudiéndose mencionar montes de chañares y caldenes, espinillos, gramíneas; y diferentes especies de aves, cánidos y dasipódidos, entre otros.

Finalmente, se registraron tres concentraciones de material arqueológico, ubicadas dos de ellas sobre la costa de la laguna y la tercera en inmediaciones de la desembocadura del arroyo.

\section{Laguna Chasicó: Sitios 1 y 2}

El sitio 1 se encuentra ubicado en una pequeña isla formada por médanos que fueron parte de una península en la costa SE de la laguna. Este sitio se relevó por primera vez en la década del' 90 , momento en el cual presentaba médanos erosionados en la línea de costa, y los materiales arqueológicos se extendían sobre la playa unos $500 \mathrm{~m}$. (Oliva 1996). En la actualidad, debido a la constante erosión hídrica y eólica, la concentración de materiales se restringe a unos $150 \mathrm{~m}$. sobre el remanente de los médanos. En este sitio, se identificaron artefactos líticos de cuarcita, arenisca y diferentes tipos de sílices, así como algunos fragmentos cerámicos.

Por su parte, el sitio 2 se localiza sobre la costa NE de la laguna. En este sector, la barranca está conformada por un médano erosionado que presenta en su parte superior montes de chañar y gramíneas. En sus primeros $100 \mathrm{~m}$. el médano se presenta como una pequeña barranca con una playa angosta; luego esta barranca aumenta su altura alcanzando los $4 \mathrm{~m}$. aproximadamente y la playa desaparece. Finalmente vuelve a descender hasta disiparse, conformando una playa de más de $5 \mathrm{~m}$. de ancho.

Cabe aclarar que la distribución de materiales arqueológicos se extiende de forma irregular por $300 \mathrm{~m}$. sobre esta costa, presentándose las mayores concentraciones en los últimos 100 $\mathrm{m}$. Se identificaron tiestos cerámicos y artefactos líticos (entre los que se hallan numerosos artefactos de molienda) confeccionados sobre sílices, cuarcitas, areniscas y riolitas. Entre los sílices pudieron observarse principalmente artefactos confeccionados sobre los denominados rodados "tehuelches o patagónicos" (Fidalgo et al. 1978). 


\section{Arroyo Chasicó: Sitio 1}

Este sitio se ubica a $4000 \mathrm{~m}$. de la desembocadura del arroyo Chasicó, sobre su margen derecha. Está localizado a $200 \mathrm{~m}$. aproximadamente del curso de agua y a unos $50 \mathrm{~m}$. de altura en relación al nivel del arroyo. Se presenta sobre un médano erosionado de forma circular, constituyendo una hoyada con un diámetro cercano a los $15 \mathrm{~m}$. En superficie se observó la presencia de artefactos líticos (tallados y de molienda) sobre calcedonia, cuarcita y riolita principalmente, no hallándose cerámica, y siendo muy escasos los rodados tehuelches.

\section{DISCUSIÓN}

La llanura adyacente al Sistema Serrano de Ventania se caracteriza por ser un espacio ecotonal con una alta biodiversidad y concentración de nutrientes básicos; con cursos y cuerpos de agua permanentes, cercanía a salinas y salitrales, y con recursos líticos localizados en determinados sectores (como las sierras y los arroyos).

Con el fin de reconocer la disponibilidad de recursos en los diferentes sectores de la llanura adyacente al Sistema Serrano de Ventania y analizar las vinculaciones establecidas entre los distintos ambientes del área, en este trabajo se consideró su subdivisión en cuenca media del arroyo Chasicó, área adyacente a dicho arroyo y cuenca inferior del arroyo Chasicó. Esto posibilitó analizar secuencialmente el uso diferencial de cada ambiente, en relación con los recursos presentes, la posibilidad de acceso a los mismos y la información disponible.

Si bien en todos los sitios presentados es recurrente la presencia de cuarcita, riolita, cuarzo y calcedonia, las estrategias de utilización de cada una de ellas varían en relación con diferentes variables, entre las que puede mencionarse las distancias que separan a cada sitio de las posibles fuentes de aprovisionamiento, calidad para la talla, formas base en las que se presentan las diferentes materias primas líticas.

En San Martín sitio 1, ubicado en la cuenca media del arroyo Chasicó se observa un importante aprovechamiento de la cuarcita en detrimento de otras materias primas. Si bien se han registrado artefactos en todos los tamaños, el 70\% está constituido por artefactos en tamaños pequeño y muy pequeño. El $25 \%$ presenta corteza entre $1-75 \%$ de su superficie, observándose algunos raspadores de sílices con corteza hasta en el $50 \%$ de su superficie. Dentro de las materias primas restantes, sólo se observan instrumentos sobre calcedonia y obsidiana; las demás materias primas están representadas únicamente por lascas de tamaños pequeños, con escasa o nula reserva de corteza. Estas particularidades también se observaron en los materiales provenientes de las excavaciones realizadas en años anteriores. Probablemente estas características se deban a la cercanía del arroyo, en el que las cuarcitas se presentan en forma de rodados, si bien no se descarta la posibilidad de que algunas de éstas hayan sido obtenidas de afloramientos primarios.

Por otro lado, en este sitio se encuentra representada la especie Adelomelon brasiliana, caracol marino localizado a lo largo de toda la costa atlántica (desde el Sur de Brasil hasta el extremo Sur de Argentina). El hallazgo de este molusco, como en el caso de San Martín sitio 1 y sitios ubicados en las márgenes de la laguna Los Chilenos y de las lagunas Encadenadas del Oeste, indica la importancia de las relaciones establecidas entre los ambientes interiores, cercanos al Sistema Serrano de Ventania y los ambientes costeros marinos, en los diferentes momentos de ocupación del área (Oliva et al. 1991a; Oliva 1996). 
Otro hallazgo destacable, es un artefacto confeccionado sobre obsidiana. Esta materia prima es recurrente en las regiones de Patagonia y Cuyo en afloramientos primarios y secundarios, encontrándose muy raramente en la Región Pampeana en forma de rodados en la costa marítima.

En el área adyacente a la cuenca del arroyo Chasicó, se consideró como caso de estudio el sitio 1 de Villa Iris. Este sitio presenta gran variabilidad de materias primas líticas, entre las que pueden mencionarse altos porcentajes de sílices (49\% de la muestra), siendo el chert silíceo el más abundante, de mayor tamaño y más formatizado (raspadores, puntas de proyectil y raederas, entre otros instrumentos). Esto se relacionaría con que este recurso podría ser considerado como local, ya que en distintos puntos de la llanura adyacente al Sistema Serrano de Ventania se han detectado depositaciones de carbonato de Calcio silicificado.

Los sílices restantes (calcedonia y demás variedades) se presentan en tamaños más pequeños, con bajo índice de reserva de corteza y en su mayoría son lascas y desechos de talla. Muchos de estos sílices podrían provenir de los llamados rodados "tehuelches o patagónicos" procedentes del Norte de Patagonia, Sur de la Región Pampeana o costa atlántica.

Por otro lado, se destaca la presencia de rodados de cuarcita muy pulidos que podrían haber sido utilizados como elementos de molienda. Algunos de ellos exhiben lascados, lo que indicaría la reutilización de los mismos, es decir el empleo de estos rodados como nódulos para la obtención de lascas.

La maximización de los recursos evidenciada en la reutilización de los rodados de cuarcita, y en el tamaño pequeño de gran parte de los artefactos recuperados, se podría atribuir a que la fuente más cercana de aprovisionamiento lítico se encuentra en el arroyo Chasicó, a $41 \mathrm{~km}$. en línea recta del sitio 1 de Villa Iris.

Otra característica observada en este sitio es el bajo índice de bifacialidad, sólo presente en ciertos instrumentos como puntas de proyectil y un raspador (unifacial pero con uno de sus filos bifacial), los cuales están confeccionados sobre chert silíceo. Esta técnica de talla no se observa en otros instrumentos confeccionados sobre otras materias primas. Además, dentro del total de la muestra se ha determinado un bajo porcentaje $(2 \%)$ de lascas producto de reducción bifacial, de las mismas una sola es de cuarcita y las demás de sílice.

Los sitios localizados en la cuenca inferior del arroyo Chasicó (sitios 1 y 2 de la Laguna Chasicó y sitio 1 del Arroyo Chasicó) se caracterizan por presentar artefactos líticos tallados así como artefactos de molienda. Entre las formas base de los primeros se encuentran los denominados "rodados tehuelches o patagónicos" y lascas de cuarcita y riolita principalmente. Las formas base observadas en los artefactos de molienda son principalmente rodados de cuarcita, provenientes del arrastre originado por los cursos de agua de esta cuenca desde los faldeos de las Sierras de Ventania.

\section{CONSIDERACIONES FINALES}

La información arqueológica procedente del sector Sudoeste de la Provincia de Buenos Aires, contribuye a la discusión sobre la dinámica de ocupación de estos territorios por parte de las sociedades cazadoras recolectoras. Los datos presentados en este trabajo en base al análisis de sitios superficiales así como de la comparación con sitios excavados, permiten 
obtener un primer acercamiento a las decisiones tomadas por estas poblaciones en relación al manejo de los recursos líticos disponibles en el área. Específicamente el caso estudiado permite proponer al Sistema de Ventania y de sus arroyos y lagunas aledañas como factores concentradores de poblaciones humanas. Así, las cuencas de los ríos y arroyos (p.ej. Arroyo Chasicó) representaron para estas poblaciones una vía natural que permitió conectar diferentes tipos de ambientes (serrano, periserrano y de llanura), en un espacio geográfico acotado (Oliva et al. 2004).

Una consideración importante se vincula con que este espacio podría ser un lugar de encuentro y/o tránsito de sociedades cazadoras recolectoras de dos áreas diferentes, el Sur de la Región Pampeana y el Norte de Patagonia, en donde estos grupos interactuaron, adquiriendo y transmitiendo diferentes tipos de decisiones en su relación con el medio. Esta dinámica entre los distintos ambientes del área (serrano, periserrano y de llanura), y a su vez con otros ambientes como la costa atlántica y el Norte de Patagonia, está evidenciada por la presencia de caracoles marinos, sílices y obsidiana. En esta movilidad, habrían jugado un papel central los cuerpos de agua, y principalmente los cauces que drenan el área, como vías de comunicación entre los distintos ambientes, donde se concentran gran diversidad de recursos críticos.

Si bien no se dispone de elementos que permitan asignar claramente a un momento cronológico en particular los sitios presentados en este trabajo, se considera en base al conjunto de información que este tipo de interacción con el ambiente se estaría dando al menos desde fines del Holoceno medio hasta tiempos de la ocupación hispánica.

\section{AGRADECIMIENTOS}

A Nora Cinquini y su familia, a María Candela Pavón "Kity” y su familia, Hugo Hernández "Rodera”. A la Asociación Cooperadora de la Reserva Natural Laguna Chasicó; al complejo pesquero "Chapalcó Ray"; a los pescadores y comunidad de Chapalcó y especialmente a los Guardaparques Fabrizio Piantanida y Daniel Porte por su asistencia permanente en las tareas de campo. A las municipalidades de Tornquist y Puan, especialmente a la Delegación de Villa Iris. La presente investigación fue posible gracias a la colaboración de la Dirección Provincial de Patrimonio Cultural del Instituto Cultural del Gobierno de la Provincia de Buenos Aires y a una Beca de Iniciación de CONICET.

Estas actividades se están llevando a cabo en el marco de los proyectos: "Investigaciones arqueológicas en el Borde Occidental de la Pampa Húmeda y Sistema Serrano de Ventania”, PIP-CONICET 2758; “El registro arqueológico del uso del espacio en ambientes ecotonales de la Región Pampeana", acreditado por la Universidad Nacional de Rosario y "Estudios Arqueológicos en las Sierras Australes de la Provincia de Buenos Aires", acreditado por la Universidad Nacional de La Plata.

\section{NOTAS}

1 La denominación sílice pampeano fue presentada por nosotros en anteriores trabajos, refiriéndonos a los depósitos de carbonato de Calcio asociados con precipitaciones de sílice. En relación con esto, el área de estudio al ser transicional entre Pampa Húmeda y Seca puede haber coadyuvado a la precipitación de sílice junto a carbonato de Calcio (San Cristóbal com. pers.). Se han identificado concreciones de carbonato de Calcio en diferentes formaciones del flanco occidental del Sistema Serrano de Ventania (De Francesco 1992). Particularmente, nuestras investigaciones permitieron detectar en cortes de caminos y borde de lagunas la presencia de precipitaciones de sílice relacionadas con carbonato de Calcio (Oliva y Avila 2001).

Cabe destacar que en el XV Congreso Nacional de Arqueología Argentina realizado en la ciudad de Río Cuarto, en septiembre de 2004, en la mesa de comunicaciones de la Región Pampeana se discutió la designación de sílice pampeano, planteándose que la misma traería confusiones en relación con su distribución regional. A partir de 
esta discusión se efectuaron nuevas consultas al Dr. Cingolani de la Facultad de Ciencias Naturales y Museo (UNLP). Dado que este tipo de registro arqueológico se limita a un área particular de la Región Pampeana se considera oportuno denominar a esta materia prima como chert silíceo, término que viene siendo utilizado por diferentes colegas de la Región Pampeana (Curtoni et al. 2004; Charlín 2002, entre otros)

2 En este trabajo se consideró pertinente evitar la utilización del término desecho para referirse a las lascas de diferentes tamaño, por no poder diferenciar en esta instancia su posible utilización. Por esta razón se optó en su reemplazo por el término genérico de lasca.

\section{BIBLIOGRAFÍA}

Aguerre, A.

2002 Cabras, soledades y médanos. La arqueología del Oeste Pampeano. En: Entre médanos y caldenes de la Pampa Seca. Aguerre, A. y A. Tapia (comp.). Facultad de Filosofía y Letras. UBA. pp.17-74.

Ameghino, F.

1910 La industria lítica del Homo pampeus procedente de la región litoral de Mar del Plata a Necochea, con presentación del material. Actas XVII Congreso Internacional de Americanistas. Buenos Aires.

Austral, A.

1968 Prehistoria del sur de la región pampeana. Actas y Memorias del 37 Congreso Internacional de Americanistas (1966), 3: 325-338. Buenos Aires.

Barrientos, G., M. Leipus y F. Oliva

1997 Investigaciones arqueológicas en la Laguna Los Chilenos. (Provincia de Buenos Aires). En: M. Berón y G. Politis (Eds.) Arqueología pampeana en la década de los '90. XI Congreso Nacional de Arqueología Argentina. Mendoza. pp. 115-126.

Barrientos, G., F. Oliva y M. Del Papa

2002 Historia pre y post depositacional y tafonómica del entierro secundario del sitio Laguna Los Chilenos 1 (Provincia de Buenos Aires). Relaciones de la Sociedad Argentina de Antropología, 27: 303-326. Buenos Aires

Bayón, C.; N. Flegenheimer; M. Valente y A. Pupio

1999 Dime cómo eres y te diré de dónde vienes: procedencia de rocas cuarcíticas en la Región Pampeana. Relaciones de la Sociedad Argentina de Antropología, 24: 237-255. Buenos Aires

Berón, M.; L. Migale y R. Curtoni

1995 Hacia la definición de una base regional de recursos líticos en el área del Curacó. Una cantera taller. Puesto Córdoba (La Pampa, Argentina). Relaciones de la Sociedad Argentina de Antropología, 20: 111-129. Buenos Aires.

Bonorino, A., E. Ruggiero y E. Mariño

1989 Caracterización hidrológica de la cuenca del arroyo Chasicó. Programa Prioritario de Asesoramiento a Municipalidades. Informe 44. CIC. La Plata. 35 pp.

Buckley, W.

1978 La epistemología vista a través de la teoría de sistemas. En: G. Klir (comp.) Tendencias en la teoría general de sistemas. Alianza Universidad. Madrid. Pp. 219-236. 
Cabrera, A.

1971 Fitogeografía de la República Argentina. Boletín de la Sociedad Argentina de Botánica, 14 $(1-2): 1-42$.

Castro, A

1983 Noticia preliminar sobre un yacimiento en la Sierra de la Ventana, Provincia de Buenos Aires. Relaciones de la Sociedad Argentina de Antropología, 15: 91-107. Buenos Aires

Charlín,J.

2002 Aprovisionamiento de materia primas líticas en el N.O. de la provincia de La Pampa a fines de siglo XIX. En: D. Mazzanti, M. Berón, M. y F. Oliva (Eds.) Del Mar a los Salitrales. Diez mil años de Historia Pampeana en el Umbral del Tercer Milenio. Pp.205-218 UNMdP. SAA.

Consens, M y F. Oliva

1999 Estado de las investigaciones en sitios con representaciones rupestres en la Región Pampeana, República Argentina. Actas del XII Congreso Nacional de Arqueología Argentina, 3: 119-127 La Plata.

Curtoni, C., P. Barros y M. Berón

2004 Meseta del Fresco: análisis de canteras y talleres. Perspectivas arqueológicas regionales. En: C. Gradin y F. Oliva (Eds.) La Región Pampeana -su pasado arqueológico-. Ed Laborde. pp 287-296.

De Francesco, F.

1992 Estratigrafía del cenozoico en el flanco occidental de las sierras de Curamalal. Sierras Australes Bonaerenses. Terceras Jornadas Geológicas Bonaerenses. La Plata.

Falkner. T.

[1774] 2003 Descripción de la Patagonia y de las partes contiguas de la América del Sur. Editorial Taurus. p. 221.

Fidalgo, F., Laza, J., Porro, N. y E. Tonni

1978 Algunas características de la Formación Arroyo Chasicó y sus relaciones geológicas. VII Congreso de Geología Argentina, 1: 213-225. Neuquén.

Frère, M. e I. González de Bonaveri

1993 La utilización de ambientes lagunares: Laguna Los lobos - Provincia de Buenos Aires. Arqueología 3: 171-197 Buenos Aires. Revista de la Sección Prehistoria, ICA-UBA.

Gradin, C.

1978 Algunos aspectos del análisis de las manifestaciones rupestres. Revista del Museo Provincia 1, 2 Neuquén.

Holmberg, E.

1884 La Sierra de Curá-Malal (Currumalan). Informe presentado al excelentísimo señor gobernador de la Provincia de Buenos Aires, Doctor Dardo Rocha. Buenos Aires. 81pp.

Hrdlicka, A.

1912 Early man in South America. Smithsonian Institute Bureau of American Ethnology, 52. Washington D.C. 
Madrazo, G.

1979 Los cazadores a larga distancia de la Región Pampeana. Prehistoria Bonaerense. pp. 1267. Olavarría.

Madrid, P. y F. Oliva

1994 Análisis preliminar de las representaciones rupestres presentes en cuatro sitios del Sistema de Ventania, Provincia de Buenos Aires. Revista del Museo de La Plata, (Nueva Serie), 9: 200-223. La Plata.

Martínez, G.

2002 Organización y cambio en las estrategias tecnológicas: Un caso arqueológico e implicaciones comportamentales para la evolución de las sociedades cazadoras recolectoras pampeanas. En: G. Martínez y J. Lanata (Eds.) Perspectivas Integradoras entre Arqueología y Evolución. Teoría, Método y Casos de Aplicación. Serie Teórica No1. INCUAPA. Olavarría. pp. 121-156.

Mazzanti, D. y C. Quintana

2001 Cueva Tixi: Cazadores y recolectores de las Sierras de Tandilia Oriental. Laboratorio de Arqueología, Universidad Nacional de Mar del Plata. Publicación especial 1. 466 pp. Menghin, O. y M. Bórmida

1950 Investigaciones prehistóricas en Cuevas de Tandilia (Provincia de Buenos Aires). Runa, 3, (1-2). Buenos Aires.

Mithen, S.

1990 Thoughtful foragers. A Study of Prehistory Decision Making. Cambridge University Press. 289 pp.

Musters, G.

[1911] 2005 Vida entre los Patagones. Editorial Elefante Blanco. Buenos Aires. 384 p.

Oliva, F.

2000 Análisis de las localizaciones de los sitios con representaciones rupestres en el Sistema de Ventania, Provincia de Buenos Aires En: M. Podestá y M. Hoyos (Eds.) Arte en las Rocas Arte Rupestre, menhires y piedras de colores en Argentina. Sociedad Argentina de Antropología. Buenos Aires pp.143-162.

Oliva, F.

1996 Investigaciones arqueológicas en el Sector Occidental de Ventania y la llanura adyacente. Beca Postdoctoral. Informe presentado al CONICET. MS.

Oliva, F. y M. Algrain

2005 Representaciones simbólicas de las sociedades indígenas en el Área Ecotonal HúmedaSeca Pampeana (AEHSP). ¿Arte shamánico?. Revista de la Escuela de Antropología, 10: 155-167. Escuela de Antropología, Facultad de Humanidades y Artes, Universidad Nacional de Rosario.

Oliva F. y M. Algrain

2004 Una aproximación cognitiva al estudio de las representaciones rupestres del Casuhati (Sistema Serrano de Ventania y llanura adyacente, Provincia de Buenos Aires) En: C. Gradin y F. Oliva (Eds.) La Región Pampeana-su pasado arqueológico-. Ed Laborde. Argentina. pp 49-60. 
Oliva, F. y J. Avila

2001 Informe sobre el Impacto sobre el registro arqueológico: Obras LOOP al NEUBA-II, Progresiva 663 a 691, Partido de Saavedra (Post-Zanjeo). LOOP al NEUBA-II, Progresiva 850 a 867 Partido de Guaminí (Pre-Zanjeo). Presentado a la Empresa Transportadora Gas del Sur. 78 p. Ms.

Oliva, F. y J. Moirano

2001 Estrategias para el estudio de la utilización de recursos líticos en el Sur de la Región Pampeana, República Argentina. Arqueología uruguaya hacia el fin del milenio, 1:521-537. Colonia del Sacramento. Uruguay.

Oliva, F., A. Gil y M. Roa

1991a Recientes investigaciones en el Sitio San Martín 1 (BU/PU/5). Partido de Puan, Provincia de Buenos Aires. Shincal. X Congreso Nacional de Arqueología Argentina, 3 (3): 135139. Catamarca.

Oliva, F.; J. Moirano y M. Saghessi.

$1991 b$ Estado de las investigaciones arqueológicas en el sitio Laguna de Puan 1. Boletín del Centro, 2: 127-138. La Plata.

Oliva, F., E. Levin, J. Moirano y D. Avila

2000 Investigaciones arqueológicas de momentos Tardíos en el Abra de Saavedra, Provincia de Buenos Aires. Libro de Resúmenes del II Congreso de Arqueología de la Región Pampeana Argentina. Mar del Plata, pp. 18-19.

Oliva, F., J. Moirano, R. Ruiz, J. Calamante, y A. Soliz

2004 Aplicación de GIS en las investigaciones arqueológicas del Sistema de Ventania, Provincia de Buenos Aires. En: C. Gradin y F. Oliva (Eds.) La Región Pampeana -su pasado arqueológico-. Ed Laborde. Argentina. pp. 91-100.

Paleo, M., M. Paez y M. Pérez Meroni

2002 Condiciones Ambientales y Ocupación Humana durante el Holoceno Tardío en el Litoral Fluvial Bonaerense. En: D. Mazzanti, M. Berón y F. Oliva (Eds.) Del Mar a los Salitrales. Diez mil años de Historia Pampeana en el Umbral del Tercer Milenio. UNMdP. SAA. pp. 365-376.

Pérez Amat, M., D. Scheines y M. Bayón.

1985 Noticia preliminar sobre hallazgos de pinturas rupestres en el establecimiento Santa Marta (Partido de Saavedra). Sapiens, 5: 86-90. Chivilcoy.

Politis, G.

1984 Investigaciones arqueológicas en el Área Interserrana Bonaerense. Etnía 32: 3-52. Olavarría.

Politis, G. y P. Madrid

2001 Arqueología Pampeana. Estado Actual y Perspectivas. En E. Berberián y A. Nielsen (Eds.) Historia Argentina Prehispánica, 2: 815-838. Córdoba.

Zeballos, E.

[1881] 2002 Viaje al País de los Araucanos. Editorial Elefante Blanco. Buenos Aires. p. 506. 


\begin{tabular}{|c|c|c|c|c|c|c|c|c|c|c|c|}
\hline & $\begin{array}{c}\text { cuarcita } \\
\text { grases } \\
\text { fese }\end{array}$ & $\begin{array}{c}\text { cuarcita } \\
\text { grams } \\
\text { mediano }\end{array}$ & $\begin{array}{c}\text { cuarcita } \\
\text { grame } \\
\text { gueso }\end{array}$ & niolita & calcedonia & \begin{tabular}{|} 
chent \\
silices
\end{tabular} & arenbea & obsidiana & cuarzo & atras & Total \\
\hline my pequedse & \begin{tabular}{|l|}
5 \\
\end{tabular} & 28 & 9 & & 2 & & & & 1 & 2 & 48 \\
\hline perpetes & 11 & 21 & 22 & 1 & 5 & 1 & & 1 & 2 & 3 & 47 \\
\hline imedicte pequeino & 4 & 15 & 9 & 2 & & & & & & & 30 \\
\hline medians grande & 1 & 3 & 8 & & & 1 & 1 & & & & 14 \\
\hline graede & & 5 & 3 & & & & & & & 1 & 9 \\
\hline my qrande & & 5 & 6 & & & & & & & & 11 \\
\hline Total & 22 & 77 & 57 & 3 & 7 & 2 & 1 & 1 & 3 & 6 & 179 \\
\hline
\end{tabular}

Tabla 1. San Martín Sitio 1. Frecuencia de Materias primas por tamaño

\begin{tabular}{|c|c|c|c|c|c|c|c|c|c|c|c|}
\hline grupo tipológico & $\begin{array}{c}\text { cuarcita } \\
\text { grano } \\
\text { fino }\end{array}$ & $\begin{array}{c}\text { cuarcita } \\
\text { grano } \\
\text { mediano }\end{array}$ & $\begin{array}{c}\text { cuarcita } \\
\text { grano } \\
\text { grueso }\end{array}$ & riolita & calcedonia & $\begin{array}{l}\text { chert } \\
\text { siliceo }\end{array}$ & arenisca & obsidiana & cuarzo & otras & Total \\
\hline ecofactos & & 9 & 35 & & & & & & & 4 & 48 \\
\hline raspadores & 1 & 1 & 1 & & 2 & & & & 1 & & 6 \\
\hline raederas & & 2 & & & & & & & & & 2 \\
\hline $\begin{array}{l}\text { punta de proyectil } \\
\text { fragmento de limbo }\end{array}$ & 1 & & & & & & & & & & 1 \\
\hline núcleos de lascas & & 2 & 1 & 1 & & & & & & & 4 \\
\hline lascas & 19 & 58 & 7 & 2 & 5 & 2 & & 1 & 1 & 2 & 97 \\
\hline $\begin{array}{l}\text { desechos no } \\
\text { clasificables }\end{array}$ & 1 & 3 & 12 & & & & & & 1 & & 17 \\
\hline $\begin{array}{l}\text { artefactos de } \\
\text { molienda }\end{array}$ & & 1 & 1 & & & & 1 & & & & 3 \\
\hline $\begin{array}{l}\text { lito manuf por } \\
\text { lascados }\end{array}$ & & 1 & & & & & & & & & 1 \\
\hline Total & 22 & 77 & 57 & 3 & 7 & 2 & 1 & 1 & 3 & 6 & 179 \\
\hline
\end{tabular}

Tabla 3. San Martín sitio 1. Frecuencia de materias primas por grupos tipológicos.

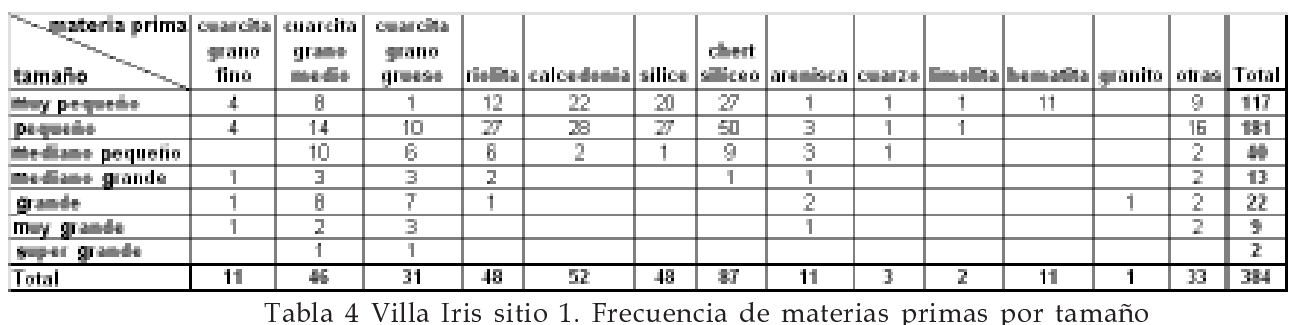

Tabla 4 Villa Iris sitio 1. Frecuencia de materias primas por tamaño 


\begin{tabular}{|c|c|c|c|c|c|c|c|c|c|c|c|c|c|c|}
\hline grupo tipologico & $\begin{array}{c}\text { crancita } \\
\text { grano } \\
\text { fino }\end{array}$ & \begin{tabular}{|c|} 
cumchis \\
grano \\
medio
\end{tabular} & \begin{tabular}{|c|} 
cuarcita \\
gramb \\
grueso
\end{tabular} & rintits & calcedonia & silice & \begin{tabular}{|c|} 
chent \\
silices
\end{tabular} & menisca & conse & limolita & bematita & granito & atras & Total \\
\hline ecufactós & & 1 & 4 & & & & 5 & \begin{tabular}{|l|}
3 \\
\end{tabular} & \begin{tabular}{|l|}
1 \\
\end{tabular} & & & & 9 & 23 \\
\hline rapolunes & & & & & 7 & 3 & 6 & & & & & & & $\mathrm{w}$ \\
\hline reedetas & & & & & & 1 & 1 & & & & & & 1 & 3 \\
\hline puntas de proyectil & & & & & & 2 & & & & & & & & 2 \\
\hline artet foumat sumaria & & 3 & & 1 & 4 & 3 & 4 & & & & & & 1 & 16 \\
\hline nuidess & 1 & 5 & 2 & & & & 1 & & & & & & 2 & 11 \\
\hline lascas & 7 & 25 & 21 & 35 & 30 & 32 & 51 & 5 & 1 & 2 & & & 15 & 226 \\
\hline lascas bipolanes & & & & & 2 & & & & & & & & & 2 \\
\hline $\begin{array}{l}\text { deseclins at } \\
\text { dadificables }\end{array}$ & 3 & 11 & 1 & 12 & 9 & 6 & 19 & 1 & 1 & & & 1 & 5 & 6) \\
\hline $\begin{array}{l}\text { artefinctos de } \\
\text { molienda. }\end{array}$ & & & 1 & & & & & 1 & & & & & & 2 \\
\hline $\begin{array}{l}\text { litos manif por } \\
\text { lascods }\end{array}$ & & & 2 & & & 1 & & & & & & & & 3 \\
\hline $\begin{array}{l}\text { pigmentos no } \\
\text { pisparados }\end{array}$ & & & & & & & & & & & 11 & & & 11 \\
\hline Total & 11 & 46 & $3 \pi$ & 48 & 52 & 48 & 87 & 11 & 3 & 2 & 11 & 1 & 33 & 384 \\
\hline
\end{tabular}

Tabla 5 Villa Iris sitio 1. Frecuencia de materias primas por grupo tipológico

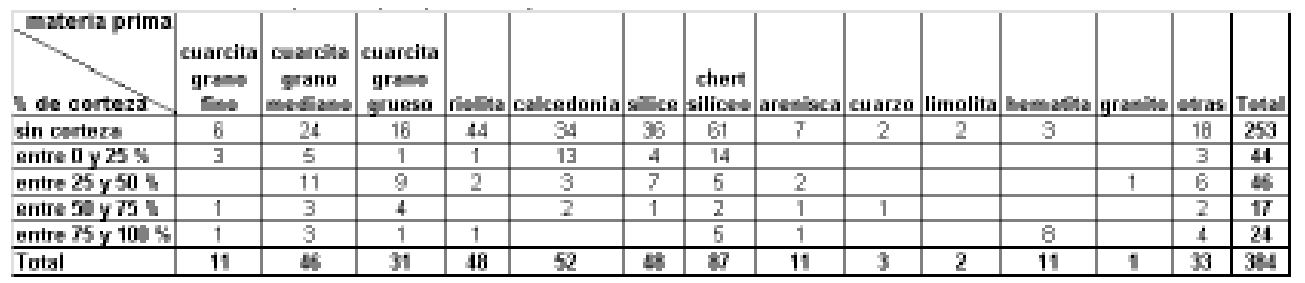

Tabla 6 Villa Iris sitio 1. Frecuencia de materias primas por porcentaje 